\title{
Klf4 inhibits tumor growth and metastasis by targeting microRNA-31 in human hepatocellular carcinoma
}

\author{
CHUAN TIAN ${ }^{1-3^{*}}$, SHANSHAN YAO ${ }^{1,3^{*}}$, LI LIU $^{4 *}$, YOUCHENG DING ${ }^{5}$, QINGWANG YE $^{6}$, \\ XIAO DONG ${ }^{1}$, YONG GAO ${ }^{3}$, NING YANG ${ }^{6}$ and $\mathrm{QI} \mathrm{LI}^{1}$ \\ ${ }^{1}$ Department of Oncology, Shanghai First People's Hospital Affiliated to Shanghai Jiaotong University, Shanghai; \\ ${ }^{2}$ Department of Nuclear Medicine, Guizhou Provincial People's Hospital, Guiyang, Guizhou; ${ }^{3}$ Department of Oncology, \\ Shanghai East Hospital, Tongji University, Shanghai; ${ }^{4}$ Department of Pharmacy, Guiyang Hospital of \\ Guizhou Aviation Industry Group, Guiyang, Guizhou; ${ }^{5}$ Department of General Surgery, Shanghai East Hospital, \\ Tongji University; ${ }^{6}$ Department of Liver Surgery, Eastern Hepatobiliary Surgery Hospital, \\ Second Military Medical University, Shanghai, P.R. China
}

Received March 23, 2016; Accepted November 18, 2016

DOI: $10.3892 / \mathrm{ijmm} .2016 .2812$

\begin{abstract}
MicroRNAs (miRNAs or miRs) are short, endogenous non-coding RNA molecules, demonstrating abnormal expression in cancer initiation and progression. In this study, we profiled 18 differentially regulated miRNAs, including miRNA-31, using miRNA array. Kruppel (or Krüppel)-like factor 4 (Klf4) is a transcription factor and putative tumor suppressor. Both were found to be significantly downregulated in liver cancer tissues and cells. However, little is known about the correlation between Klf4 and miRNA-31 in hepatocellular carcinoma (HCC). The mRNA expression of Klf4 was decreased and inversely associated with the clinical stage, $\mathrm{T}$ classification and hepatitis $\mathrm{B}$ in patients with HCC, while the expression of miR-31 was lower $(\mathrm{r}=0.326, \mathrm{P}=0.018)$. Using cell counting kit 8 (CCK8) and Transwell migration assays, we found that Klf4 and miR-31 inhibited the proliferation and metastasis of liver cancer cells. Moreover, we demonstrated that Klf4 directly binds to the promoter of miR-31 and activates its transcription. In vitro experiments confirmed that Klf4 regulated miR-31 and thereby inhibited HCC cell growth and metastasis. Taken together, our findings indicate that Klf4
\end{abstract}

Correspondence to: Professor Qi Li, Department of Oncology, Shanghai First People's Hospital Affiliated to Shanghai Jiaotong University, 100 Haining Road, Hongkou, Shanghai 200080, P.R. China E-mail: leeqi2001@hotmail.com

Professor Ning Yang, Department of Liver Surgery, Eastern Hepatobiliary Surgery Hospital, Second Military Medical University, 225 Changhai Road, Shanghai 200438, P.R. China

E-mail: lancet00@163.com

${ }^{*}$ Contributed equally

Key words: Kruppel-like factor 4, microRNA-31, proliferation, metastasis, hepatocellular carcinoma directly regulates miR-31 in HCC. Thus, miR-31 may serve as a potential diagnostic marker and therapeutic target in HCC.

\section{Introduction}

Hepatocellular carcinoma (HCC) is one of the most frequent and most lethal types of cancer worldwide with hardly any effective treatment available thus far (1). The major risk factors for the disease vary, including hepatitis B or $\mathrm{C}$ virus infection, alcoholic liver disease, and non-alcoholic fatty liver disease. There is still no systemic treatment available to effectively treat HCC (2). Accumulating evidence suggests that the loss of certain tumor suppressors and the aberrant regulation of cellular growth signaling, such as the extracellular signal-regulated kinase (ERK)/mitogen-activated protein kinase (MAPK) pathway and $\mathrm{Wnt} / \beta$-catenin pathway, are associated with liver tumorigenesis; however, the definite molecular pathogenesis of HCC remains poorly understood (3). Recently, the aberrant regulation of non-coding RNAs (ncRNAs) has been proposed to be associated with hepatocarcinogenesis. These ncRNAs can be further subdivided into two major categories, long non-coding RNAs and short non-coding RNAs (4). To date, the most extensively studied small RNAs in cancer are the microRNAs (miRNAs or miRs).

miRNAs are short non-coding RNAs, approximately 21-25 nucleotides in length, which target mRNAs for degradation or translational repression by direct binding to their 3'-untranslated region (3'-UTR) (5). The involvement of miRNAs in cancer pathogenesis is well established, as miRNAs can function as either oncogenes or tumor suppressor genes, depending on the cellular function of their targets. Moreover, the activation or suppression of specific miRNAs is established through oncogenes, such as myc, or tumor suppressor genes, such as p53, which can induce or inhibit tumorigenesis. Although their functions remain elusive, it is evident that miRNAs play an important role in cancer initiation and progression via the regulation of tumor suppressor genes or oncogenes (6). Previous studies have now linked the deregula- 
tion of miRNA expression to the development of liver cancer. The downregulation of miRNAs is observed in liver cancer and the target genes regulated by these miRNAs possibly act as oncogenes. On the contrary, overexpressed miRNAs may have oncogenic functions by targeting tumor suppressors in HCC. For instance, some miRNAs, such as miR-29, miR-21 and miR-221, have been reported to regulate tumor cell growth, apoptosis, migration and invasion by targeting proteins involved in those cellular pathways (7-9). However, the roles of miRNAs in hepatic carcinogenesis are complex; different studies have reported unique profiles, with only a few miRNAs in common, indicating the heterogeneity of HCC. For example, it has been shown that miR-26a inhibits (10), whereas miR-17-5p promotes tumor growth and metastasis in HCC (11).

miR-31 functions as an antitumor miRNA and presents distinct expression patterns in different human cancers. miR-31 has been found to be significantly overexpressed in colorectal cancer (12), squamous cell carcinoma of the tongue (13), head and neck squamous cell carcinoma (14), lung cancer (15) and esophageal squamous cell carcinoma (16); it has been found to be downregulated in bladder tumors (17), prostate carcinoma (18), gastric cancer (19), HCC (20), breast cancer (21) and serous ovarian carcinoma (22). Studies have suggested that Kruppel (or Krüppel)-like factor 4 (Klf4), a zinc-finger transcription factor, functions as a tumor suppressor or an oncogene. It plays an important role in cell proliferation and metastasis by regulating the expression of a number of downstream target genes (23). Accumulating clinical, experimental and mechanistic evidence suggests that Klf4 functions as a tumor suppressor in various types of cancer, including HCC (24-27). However, the mechanistic role of Klf4 in HCC and its causal link to altered miR-31 function remain undetermined to date.

In this study, we demonstrated that the loss of Klf4 and miR-31 are evident in human HCC, and the altered expression and function of Klf4 and miR-31 contribute to liver carcinogenesis. In the present study, we profiled 18 differentially regulated miRNAs, one of these being miR-31 and aimed to determine the correlation between Klf4 and miR-31 in HCC. miR-31 may serve as a potential diagnostic marker and provide new insight into the pathogenesis of $\mathrm{HCC}$.

\section{Materials and methods}

Patients and tissue specimens. Fresh liver cancer tissue samples and neighboring non-cancerous liver tissue samples were obtained from 52 patients who underwent primary surgical resection of liver tumors at the Shanghai Eastern Hepatobiliary Surgery Hospital affiliated to the Second Military Medical University (Shanghai, China) between March and September 2015. Non-cancerous tissues were obtained $5-10 \mathrm{~cm}$ away from the primary tumor. Follwoing resection, all the samples were snap-frozen in liquid nitrogen and stored at $-80^{\circ} \mathrm{C}$ prior to RNA extraction. Patients who received preoperative treatment were excluded from the study. All included patients provided written informed consent prior to enrollment. The protocols involving human samples were conducted in conformity with the ethical principles of research and approved by the Human Resources Ethics Committee of Shanghai East Hospital affiliated to Tongji University (Shanghai, China). The histopathological diagnosis of all samples was respectively verified by two pathologists. The clinical staging was based on the 7th edition of the AJCC Cancer Staging Manual. The main demographic and clinicopathological characteristics of the patients are presented in Table I.

Cell lines and culture. The human HCC cell lines, Bel-7402, Sk-hep-1, Hep3B, Huh7 and HepG2, were obtained from the Shanghai Cell Bank of Chinese Academy of Sciences (Shanghai, China). The liver cell line, L02, was purchased from Li Yandong Research Group Shanghai East Hospital affiliated to Tongji University School of Medicine.

Each cell line was cultured in minimum essential medium (MEM) supplemented with $10 \%$ fetal bovine serum (FBS; HyClone, Logan, UT, USA) as well as $100 \mathrm{U} /$ $\mathrm{ml}$ penicillin and $100 \mu \mathrm{g} / \mathrm{ml}$ streptomycin. All the abovementioned cells were maintained in a humidified incubator at $37^{\circ} \mathrm{C}$ with $5 \% \mathrm{CO}_{2}$.

Western blot analysis. Protein was extracted from the cells and tissues using RIPA lysis buffer [1\% NP40, 0.1\% sodium dodecyl sulfate (SDS), $100 \mu \mathrm{g} / \mathrm{ml}$ phenylmethylsulfonyl fluoride, $0.5 \%$ sodium deoxycholate, in PBS] on ice. The supernatants were collected following centrifugation at $12,000 \mathrm{x} \mathrm{g}$ at $4^{\circ} \mathrm{C}$ for $20 \mathrm{~min}$. The protein concentration was determined using a BCA protein assay kit (Bio-Rad, Shanghai, China), and whole lysates were mixed with 4 X SDS loading buffer [125 mmol/1 Tris-HCl, 4\% SDS, 20\% glycerol, $100 \mathrm{mmol} / 1$ dithiothreitol (DTT) and $0.2 \%$ bromophenol blue] at a ratio of $1: 3$. The samples were heated at $100^{\circ} \mathrm{C}$ for $5 \mathrm{~min}$ and were separated on SDS-polyacrylamide gels. The separated proteins were then transferred onto PVDF membranes (Millipore, Bedford, MA, USA). The membranes were first probed with a primary antibody. After blocking with 5\% skim milk for $2 \mathrm{~h}$, the membranes incubated with primary antibodies [rabbit anti-KLF4 (1:500, Santa Cruz Biotechnology, Santa Cruz, CA, USA). The secondary antibody was mouse anti-GAPDH (1:3000, Santa Cruz Biotechnology).

RNA extraction and reverse transcription-quantitative $P C R$ (RT-qPCR). Total RNA was isolated from the tissue samples and cultured cells using TRIzol reagent (Invitrogen, Carlsbad, CA, USA) according to the manufacturer's insstructions. The concentration of extracted RNA was measured using a NanoDrop ND-1000 Spectrophotometer (Agilent, Santa Clara, CA, USA). RNA was reverse transcribed into complementary DNA (cDNA) using the PrimeScript RT reagent kit with gDNA Eraser (Takara, Dalian, China). Quantitative PCR (qPCR) was performed using the SYBR PrimeScript RT-PCR kit (Takara, Shiga, Japan) and the ABI 7500 System (Applied Biosystems, Foster City, CA, USA) according to manufacturer's instructions. The relative expression was calculated via the comparative cycle threshold (CT) method and was normalized to the expression of U6 small RNA. The primers used are listed in Table II. The differential expression level was calculated using the $2^{-\Delta \Delta C t}$ formula. All the experiments were conducted at least 3 times.

Plasmids, small interfering RNAs, miRNAs and transfection. The miR-31 mimic, inhibitor, Klf4 siRNA and negative controls (mimic NC or inhibitor NC) were synthesized 
Table I. Correlation between the clinicopathologic characteristics of the patients with hepatocellular carcinoma and the expression of Klf4 and miR-31.

\begin{tabular}{|c|c|c|c|c|c|c|c|}
\hline \multirow[b]{2}{*}{ Characteristics } & \multirow[b]{2}{*}{$\mathrm{n}$} & \multicolumn{3}{|c|}{ Klf4 } & \multicolumn{3}{|c|}{ miR-31 } \\
\hline & & High, n (\%) & Low, n (\%) & P-value & High, n (\%) & Low, n (\%) & P-value \\
\hline \multicolumn{8}{|l|}{ Gender } \\
\hline Male & 44 & $19(43.2)$ & $25(56.8)$ & \multirow[t]{2}{*}{0.100} & $13(29.5)$ & $31(70.1)$ & \multirow[t]{2}{*}{0.072} \\
\hline Female & 8 & $1(12.5)$ & $7(87.5)$ & & $5(62.5)$ & $3(37.5)$ & \\
\hline \multicolumn{8}{|l|}{ Age (years) } \\
\hline$\geq 60$ & 13 & $5(38.5)$ & $8(61.5)$ & \multirow[t]{2}{*}{1.000} & $3(23.1)$ & $10(76.9)$ & \multirow[t]{2}{*}{0.016} \\
\hline$<60$ & 39 & $15(38.5)$ & $24(61.5)$ & & $24(61.5)$ & $15(38.5)$ & \\
\hline \multicolumn{8}{|l|}{ Differentiation } \\
\hline Poorly & 45 & $18(40.0)$ & $27(60.0)$ & \multirow[t]{2}{*}{0.563} & $14(31.1)$ & $31(68.9)$ & \multirow[t]{2}{*}{0.178} \\
\hline Moderate/well & 7 & $2(28.6)$ & $5(71.4)$ & & $4(57.1)$ & $3(42.9)$ & \\
\hline \multicolumn{8}{|l|}{ Clinical stage } \\
\hline I-II & 14 & $9(64.3)$ & $5(35.7)$ & \multirow[t]{2}{*}{0.020} & $9(64.3)$ & $3(21.4)$ & \multirow[t]{2}{*}{0.009} \\
\hline III-IV & 38 & $11(28.9)$ & $27(71.1)$ & & $13(34.2)$ & $27(71.1)$ & \\
\hline \multicolumn{8}{|l|}{$\mathrm{T}$ classification } \\
\hline T1-T2 & 11 & $8(72.7)$ & $3(27.3)$ & \multirow[t]{2}{*}{0.029} & $4(36.4)$ & $7(63.6)$ & \multirow[t]{2}{*}{0.535} \\
\hline T3-T4 & 41 & $15(36.6)$ & $26(63.4)$ & & $11(26.8)$ & $30(73.2)$ & \\
\hline \multicolumn{8}{|l|}{ Hepatitis B } \\
\hline Negative & 6 & $4(66.7)$ & $2(33.4)$ & \multirow[t]{2}{*}{0.043} & $3(50.0)$ & $3(50.0)$ & \multirow[t]{2}{*}{0.400} \\
\hline Positive & 46 & $12(26.1)$ & $34(73.9)$ & & $15(32.6)$ & $31(67.4)$ & \\
\hline \multicolumn{8}{|c|}{ Pathological types } \\
\hline $\mathrm{HCC}$ & 38 & $16(42.1)$ & $22(57.9)$ & \multirow[t]{2}{*}{0.374} & $11(28.9)$ & $27(71.1)$ & \multirow[t]{2}{*}{0.157} \\
\hline Other types & 14 & $4(28.6)$ & $10(71.4)$ & & $7(50.0)$ & $7(50.0)$ & \\
\hline \multicolumn{8}{|l|}{ Tumor size } \\
\hline$\geq 6 \mathrm{~cm}$ & 47 & $17(36.2)$ & $30(63.8)$ & \multirow[t]{2}{*}{0.298} & $16(34.0)$ & $31(66.0)$ & \multirow[t]{2}{*}{0.790} \\
\hline$<6 \mathrm{~cm}$ & 5 & $3(60.0)$ & $2(40.0)$ & & $2(40.0)$ & $3(60.0)$ & \\
\hline
\end{tabular}

Klf4, Kruppel-like factor 4.

by GenePharma, Shanghaim, China. The previously described plasmid Klf4 and the empty control vector plasmid were obtained from Xie Keping Research Group Shanghai East Hospital affiliated to Tongji University School of Medicine. The transfection of the plasmids and siRNAs, respectively, into the HCC cells was performed using Lipofectamine 2000 (Invitrogen) transfection reagent. The relative levels of Klf4 and miR-31 in the transfected cells were examined by RT-qPCR. The cells were transfected with the plasmids or siRNAs at various concentrations as indicated $48 \mathrm{~h}$ before the performance of functional assays.

NanoString. A total of $2 \times 10^{6}$ Hep3B cells grown in the exponential phase were seeded in 6-well plates and cultured for 2 days. The cells were then harvested for total RNA using the miRNeasy kit (Qiagen). A total of $100 \mathrm{ng}$ of total RNA was assayed using the Human nCounter miRNA Assay $2.0 \mathrm{kit}$ following the manufacturer's instructions (NanoString Technologies, Seattle, WA, USA). Differences in miRNA expression were analyzed using the NanoSTRIDE software program with default settings. Clustering of the differentially expressed genes and heatmap generation was performed using the GenePattern Server (genepattern.broadinstitute.org). The volcano plot displaying the significance of the miRNA differences was produced using $\mathrm{R}$ version 3.0.2.

Luciferase reporter assays. For the binding of Klf4 to the miR-31 promoter, the $1.5 \mathrm{~kb}$ region directly upstream of miR-31 transcription binding site was amplified by PCR and inserted into the pGL3 vector (Promega, San Luis Obispo, CA, USA). The Hep3B and Sk-hep-1 cells were co-transfected with either pcDNA3.1 (Promega) or the pcDNA3.1-K1f4 and miR-31 promoter. At $48 \mathrm{~h}$ after transfection, luciferase activity was analyzed according to the Dual-Luciferase reporter assay system (Promega), using a GloMax fluorescence reader (Promega).

Cell proliferation assay. The Sk-hep-1 and Bel-7402 cells were transfected with the indicated miR-31 mimic or inhibitor (50 $\mathrm{nM})$, the Klf4 overexpression plasmid or and Klf4 siRNA (siKlf4; $120 \mathrm{ng}$ ), plated in 96-well plates at 
Table II. Primer sequences used in this study.

\begin{tabular}{ll}
\hline Primer name & \multicolumn{1}{c}{ Primer sequences $\left(5^{\prime} \rightarrow 3^{\prime}\right)$} \\
\hline U6-OF & CTCGCTTCGGCAGCACA \\
U6-OR & AACGCTTCACGAATTGCGT \\
Klf4-OF & ACCCTGGGTCTTGAGGAAGT \\
Klf4-OR & CATGAGCTCTTGGTAATGGAGC \\
Klf4-siKlf4 & AUCGUUGAACUCCUCGGUCUCUCUC \\
Pri-miR-31-OF & GAACTACCCACAAACCTCCTG \\
Pri-miR-31-OR & GGTGTGTCCAAGGAATAGCCA \\
miR-31-OF & TAATACTGCCTGGTAATGATGA \\
miRNA-OR & GTCGTATCCAGTGCAGGGTCCGAGGTATTCGCACTGGATACGACAGCTAT \\
miR-31-promoter(-1069To+1)-F(MluI) & CCGGACGCGTGCACAAAAGTTATACATAATGTCATTATTCTTATG \\
miR-31-promoter(-1069To+1)-R(HindIII) & GCCCAAGCTTCAGTTCCAAGTTACAGGAGAATACTATGA \\
Klf4-miR-31-mut-F1 & AATTAATGAGTGTGTTTACGCACGCACAGGTGAAAGGAAAAA \\
Klf4-miR-31-mut-R1 & TTTTCCTTCACCTGTGCGTGCGTAAACACACTCATTAATT \\
Klf4-miR-31-mut-F2 & AGTTGCTTGAGAGGCTACGCTCGCACGATTCACCTGAGCCTG \\
Klf4-miR-31-mut-R2 & CAGGCTCAGGTGAATCGTGCGAGCGTAGCCTCTCAAGCAACT \\
Klf4-miR-31-mut-F3 & GAGCATTATTTTTGGCACGCCTTCGCGGCTCATGCCTGTAAT \\
Klf4-miR-31-mut-R3 & ATTACAGGCATGAGCCGCGAAGGCGTGCCAAAAATAATGCTC \\
Negative control sense & UUCUCCGAACGUGUCACGUTT \\
Negative control antisense & ACGUGACACGUUCGGAGAATT \\
Hsa-miR-31 mimics sense & AGGCAAGAUGCUGGCAUAGCU \\
Hsa-miR-31 mimics antisense & CUAUGCCAGCAUCUUGCCUUU \\
MircoRNA inhibitor NC & CAGUACUUUUGUGUAGUACAA \\
Hsa-miR-31 inhibitors & AGCUAUGCCAGCAUCUUGCCU \\
\hline
\end{tabular}

Mutant sequences are underlined.

3,000 cells/well and maintained in culture medium. They were assessed at days 1-6 of culture with $0.5 \mathrm{mg} / \mathrm{ml}$ cell counting kit 8 (CCK8; Dojindo Laboratories, Kumamoto, Japan) at $37^{\circ} \mathrm{C}$ for $3 \mathrm{~h}$ and then shaken for $20 \mathrm{~min}$. The optical density was determined at $450 \mathrm{~nm}$ using an enzyme-linked immunosorbent assay reader (Dasit, Milan, Italy). Each experiment was conducted for at least 3 times and the average of the results was analyzed.

Cell migration assay. The transfected HCC cells $\left(5 \times 10^{4}\right)$ in serum-free medium were placed into the upper part of a Transwell chamber in a 24-well format with $8 \mathrm{~mm}$ diameters (Corning, Corning, NY, USA). In the bottom chamber, $800 \mu 1$ of normal MEM medium containing $10 \%$ FBS were added as a chemoattractant and the chambers were incubated for $24-48 \mathrm{~h}$ at $37^{\circ} \mathrm{C}$ and $5 \% \mathrm{CO}_{2}$. The cells on the upper part of the Transwell chamber were removed using a cotton swap, and the cells that had migrated through the membrane were stained with $0.05 \%$ crystal violet for $2 \mathrm{~h}$. Finally, the migrated cells were counted in 5 random fields under a microscope (Olympus, Shanghai, China) and the average number of 5 fields was calculated. All assays were performed in triplicate and repeated 3 times.

Wound healing assay. For the wound healing assay, $3.5 \times 10^{5}$ of examined HCC cells were seeded into 6-well plates to achieve $90 \%$ confluence. Wounds were produced in confluent mono- layer cells using a plastic tip, the cell debris were removed using phosphate-buffered saline (PBS), and $0.5 \%$ FBS-containing MEM was added. The scratched cells were then incubated at $37^{\circ} \mathrm{C}$ with $5 \% \mathrm{CO}_{2}$. The initial scratched gap breadth $(0 \mathrm{~h})$ and the residual scratched gap breadth $(48 \mathrm{~h})$ were measured using a light microscope (Nikon, Tokyo, Japan).

Statistical analyses. Quantitative data are presented as the means \pm SEM. Parameters of two-tailed, 95\% CI were used for statistical analysis. Only P-values $<0.05$ were considered to indicate statistically significant differences.

\section{Results}

miRNAs are differentially expressed in the Hep3B cells transfected with the empty vector or the Klf4 overexpression plasmid. To identify candidate miRNAs that may play a role in HCC, RT-qPCR and western blot analysis of Klf4 expression in the Hep3B cells transfected with the Klf4 plasmid were initially performed (Fig. 1A). We found that the mRNA and protein expression of Klf4 was significantly increased following the overexpression of Klf4. We then compared miRNA expression levels between the empty vector- and Klf4 overexpression vector-transfected Hep3B cells using the nCounter NanoString platform. Using RNA derived from the Hep3B cells transfected with the empty vector or Klf4 

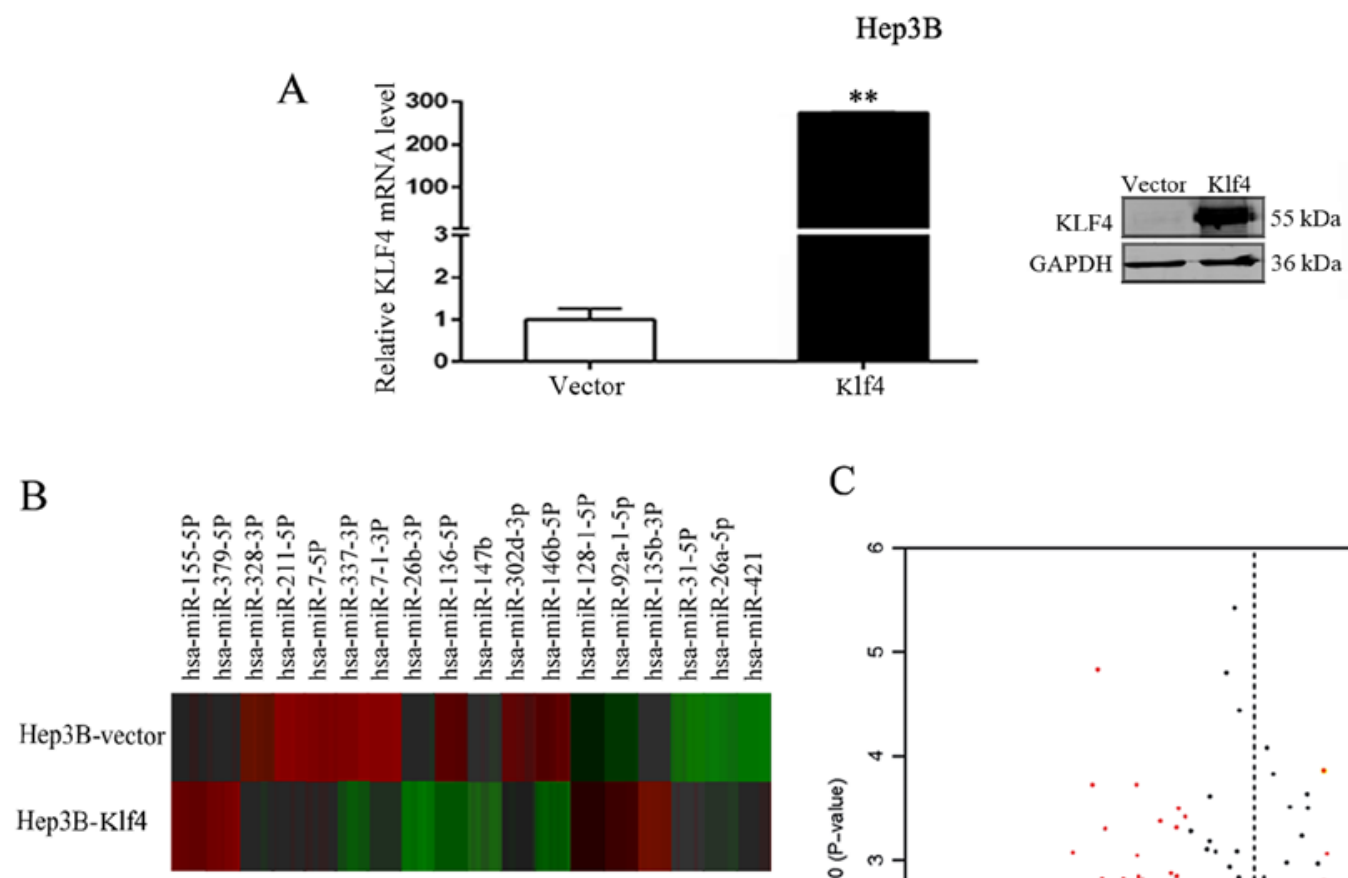

C

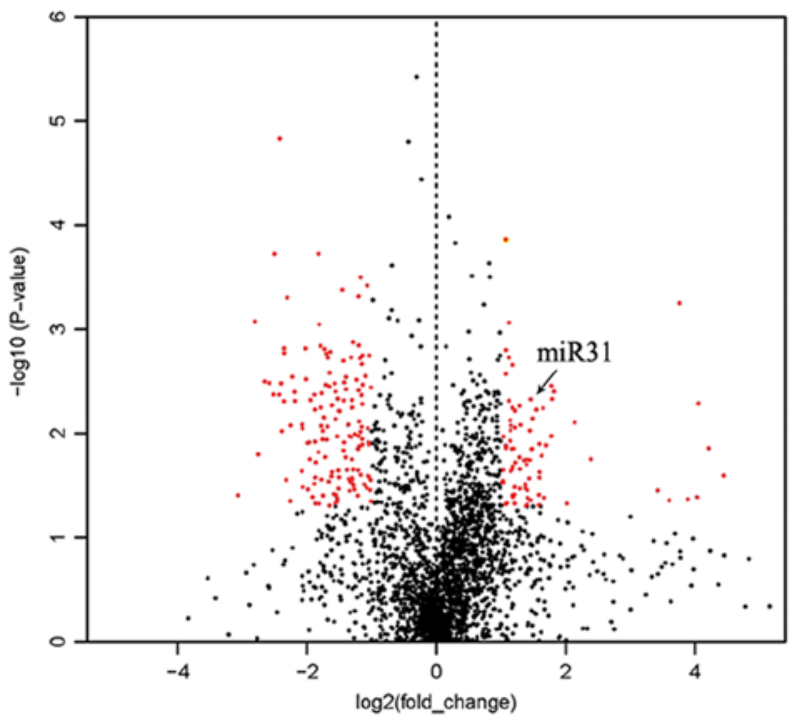

Figure 1. Differentially expressed miRNAs in Hep3B cells transfected with empty vector (Hep3B-vector) or with Klf4 overexpression vector (Hep3B-Klf4). (A) RT-qPCR and western blot analysis of Klf4 expression in Hep3B cells transfected with Klf4 plasmid. "* P<0.01. (B) Heatmap of 18 most differentially regulated miRNAs from the miRNA array of Hep3B cells transfected with empty vector or with Klf4 overexpression vector. There were 8 upregulated and 10 downregulated miRNAs. (C) Volcano plot demonstrating the profile of the differentially expressed miRNAs in Hep3B cells. This plot demonstrates the fold change (x-axis) and significance level expressed as the $\log _{10} \mathrm{P}$-value (y-axis). The red dots on the left represent the downregulated miRNAs in the Hep3B cells transfected with the Klf4 overexpression vector compared with the cells transfected with the empty vector. The red dots on the right represent the upregulated miRNAs in the Hep3B cells transfected with the Klf4 overexpression vector compared with the cells transfected with the empty vector. The black dots indicate miRNAs that did not exhibit a significant change in expression. Significance was determined with a P-value cut-off of 0.05 and a 1.5 -fold change.

overexpression vector under standard culture conditions, we found that a number of miRNAs were differentially expressed. Of the 2,565 miRNAs assayed by this method, a significant difference in expression was observed in 436 miRNAs $(17.0 \%$; $\mathrm{P}<0.05,1.5$-fold), including 216 that were downregulated and 220 that were upregulated in the Hep3B cells transfected with the empty vector or Klf4 overexpression vector. The heatmap identified the 18 most differentially regulated miRNAs from the miRNA array of Hep3B cells transfected with the empty vector or Klf4 overexpression vector, including 8 upregulated and 10 downregulated miRNAs (Fig. 1B). We found that the miR-31 cluster was significantly upregulated in the Hep3B cells (fold change, 1.92; P-value, 0.009). At last, Volcano plot revealed the profile of the differentially expressed miRNAs in the Hep3B cells. This plot outlines the fold change ( $\mathrm{x}$-axis) and significance level of expression as the $\log _{10} \mathrm{P}$-value (y-axis). The red dots on the left side represent the downregulated miRNAs in the Hep3B cells transfected with the Klf4 overexpression vector compared to the cells transfected with the empty vector; the red dots on the right side represent the upregulated miRNAs in the Hep3B cells transfected with the Klf4 overexpression plasmid compared with the cells transfected with the empty vector. The black dots indicate miRNAs which did not exhibit a significant change in expression. Significance was determined with a P-value cut off of 0.05 and a 1.5 -fold change (Fig. 1C). We found that miR-31 (the red dots on the right) was significantly upregulated in the Hep3B cells.

Klf4 and miR-31 are downregulated in HCC tissues. In this study, we explored the relative expression levels of Klf4 and miR-31 in 52 pairs of HCC tissues and neighboring noncancerous liver tissues by RT-qPCR. The expression of Klf4 and miR-31 was significantly downregulated in the cancerous compared to the non-cancerous tissues (Fig. 2A and B). The downregulation of Klf4 was found to inversely correlate with the clinical stage, $\mathrm{T}$ classification and hepatitis B infection, whereas the downregulation of miR-31 was directly associated with the clinical stage in patients with HCC (Table I). Of note, the decreased expression of miR-31 in HCC was observed in $65.4 \%(34 / 52)$ of the cases (Fig. 2C). Additionally, the endoge- 

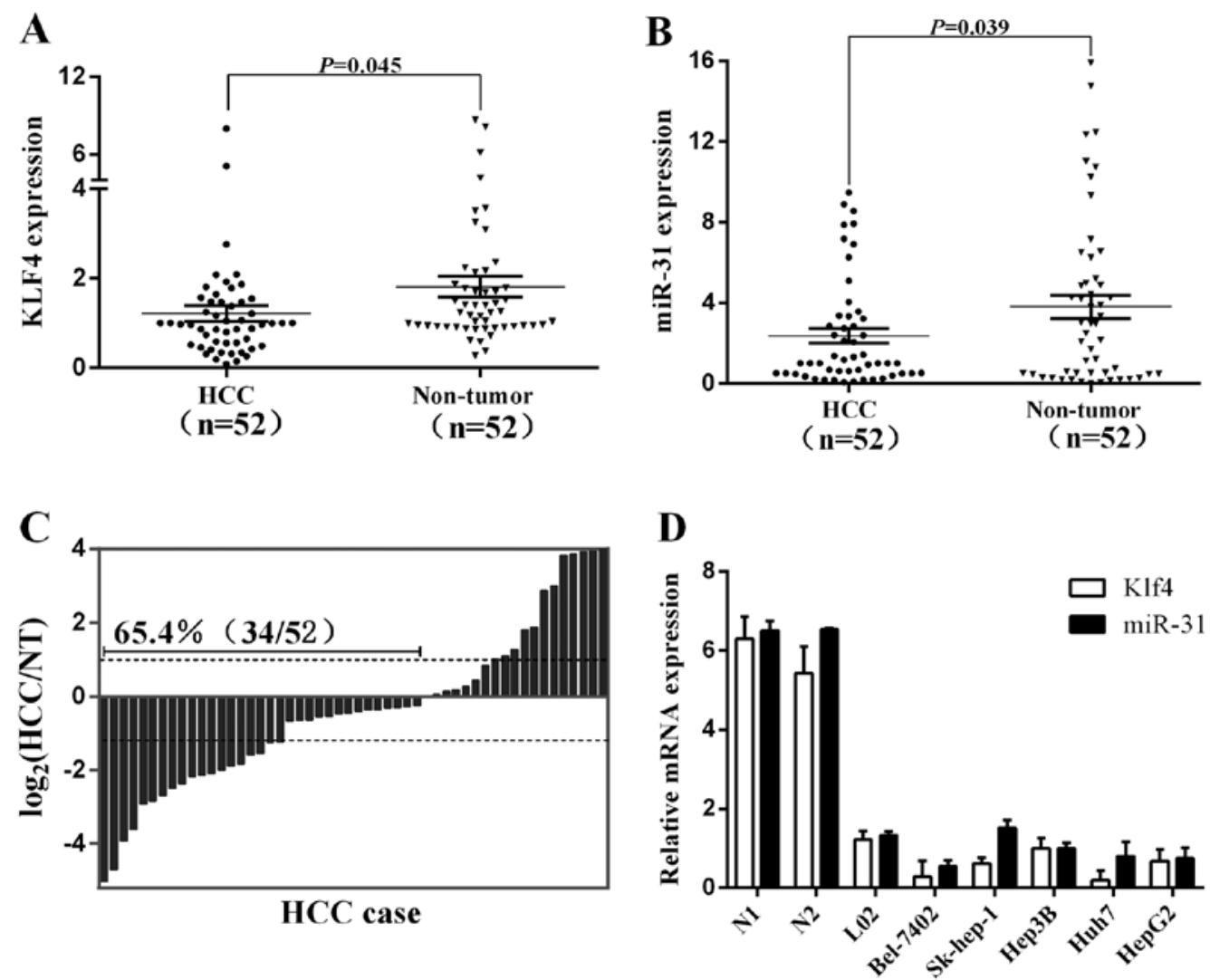

Figure 2. Kruppel-like factor 4 (Klf4) and miR-31 are downregulated in hepatocellular carcinoma (HCC). (A and B) Klf4 and miR-31 expression was significantly decreased in HCC compared to the corresponding non-tumorous (NT) liver tissues as shown by RT-qPCR. (C) miR-31 expression was significantly decreased in HCC compared to the corresponding NT livers as shown by RT-qPCR. Expression is shown as a $\log _{2}$-fold change. (D) Klf4 and miR-31 expression in 2 normal liver tissues, the immortalized liver cell line L02 and HCC cell lines. Klf4 and miR-31 expression was lower in HCC cell lines compared to normal liver (N1). Data are the means \pm SEM.

nous expression of Klf4 and miR-31 was analyzed by qRT-PCR in six chosen human liver cell lines, including an immortalized normal hepatic cell line (Fig. 2D). The liver cancer cell lines (Bel-7402, Sk-hep-1, Hep3B, Huh7 and HepG2) exhibited relatively low Klf4 and miR-31 expression levels compared to the non-cancer cell line, L02. These findings again suggest that the expression of Klf4 and miR-31 is suppressed in HCC and that a low expression of Klf4 and miR-31 is associated with the biological process of tumorigenesis in HCC.

miR-31 is directly regulated by the transcription factor Klf4. The expression of Klf4 in HCC was examined by RT-qPCR and western blot analysis. The Klf4 mRNA and protein levels were significantly increased when the Sk-hep-1 and Bel-7402 cells were transfected with the Klf4 overexpression plasmid (Fig. 3A). We then examined the expression of miR-31 by RT-qPCR, and found that it was significantly increased and decreased when the Hep3B, Bel-7402 and Sk-hep-1 cells were transfected with the Klf4 overexpression plasmid and or siKlf4, respectively (Fig. 3B-D). Moreover we examined the pri-miR-31 levels by RT-qPCR, and the results revealed that the levels were significantly increased and decreased when the Hep3B, Bel-7402 and Sk-hep-1 cells were transfected with the Klf4 overexpression plasmid and siKlf4, respectively (Fig. 3E-G).

To explore this regulation of miR-31 expression, we analyzed the $1.5 \mathrm{~kb}$ region of miR-31 upstream and found 3 binding sites of Klf4 (mut1-3). We then examined the effects of the transcription factor Klf4 on miR-31 promoter-driven luciferase activity in Hep3B cells (Fig. $3 \mathrm{H}$ ). The mutation of the Klf4 binding site 3 (mut3) abolished the effects of Klf4 on the relative luciferase activity in the Hep3B and Sk-hep-1 cells, while mutations of other binding sites had no significant effect (Fig. 3I and J). Thus, Klf4 regulates the expression of miR-31 by binding to site 3 , indicating that Klf4 is involved in the transcriptional regulation of miR-31 by directly binding to the miR-31 promoter. A positive correlation of Klf4 and miR-31 was also revealed in the HCC tissues (Fig. 3K). Taken together, these findings suggest that the transcription factor Klf4 is a functional regulator of miR-31 in HCC.

Klf4 and miR-31 inhibit HCC cell proliferation and migration in vitro. To ascertain the biological functions of Klf4 and miR-31 in HCC, following the ectopic expression of Klf4 and miR-31, we examined the growth rate of two different liver cancer cell lines, Sk-hep-1 and Bel-7402, by CCK8 assay. Transfection with siKlf4 promoted Sk-hep-1 cell proliferation, and transfection with miR-31 mimic attenuated the growth-promoting effects induced by transfection with siKlf4 (Fig. 4A). Klf4 overexpression suppressed Bel-7402 cell proliferation, while transfection with miR-31 inhibitor attenuated the growth inhibitory effects of Klf4 (Fig. 4B). The Sk-hep-1 and Bel-7402 cells were seeded onto 96-well plates and respectively transfected with Klf4 or siKlf4, miR-31 mimic or inhibitor for 1-6 days. Cell viabilities were determined by CCK8 assays. Cell growth was measured 
A

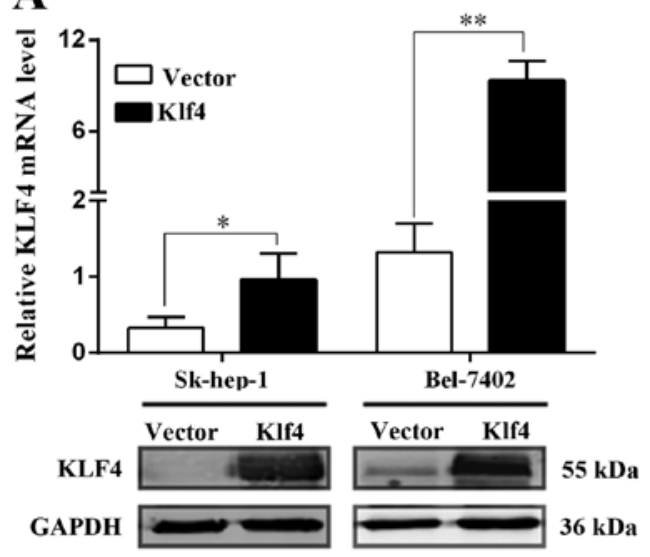

C

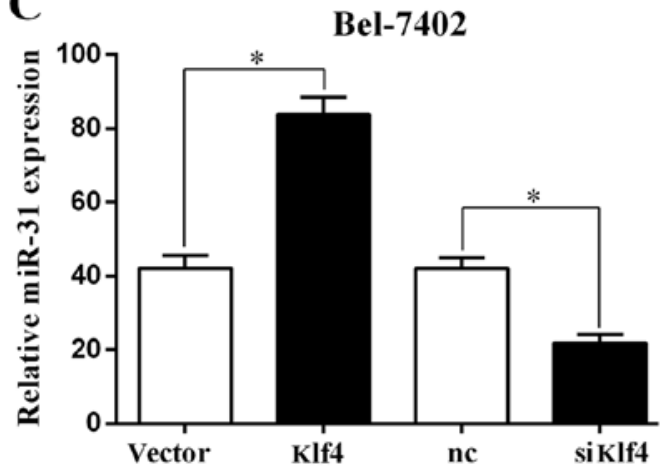

E

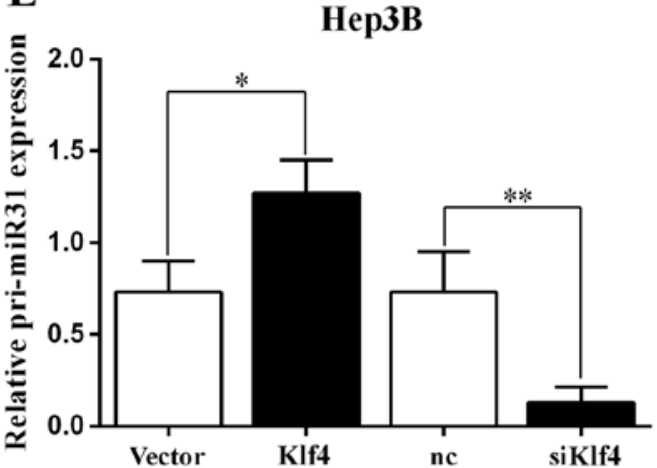

G

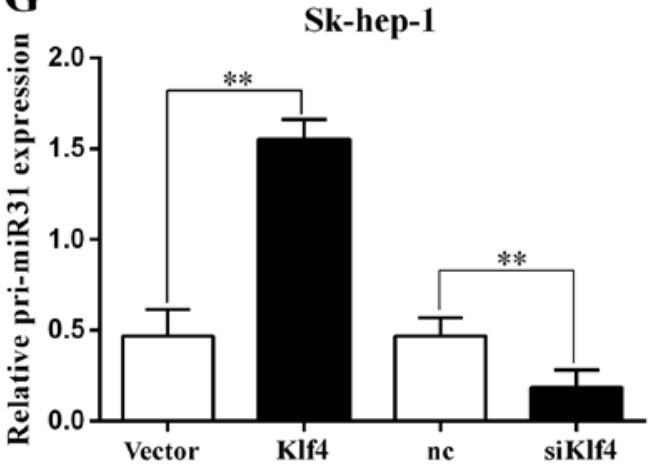

B

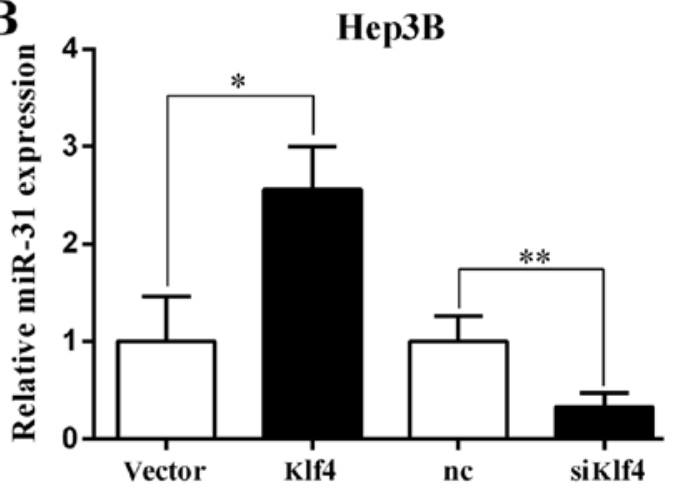

D

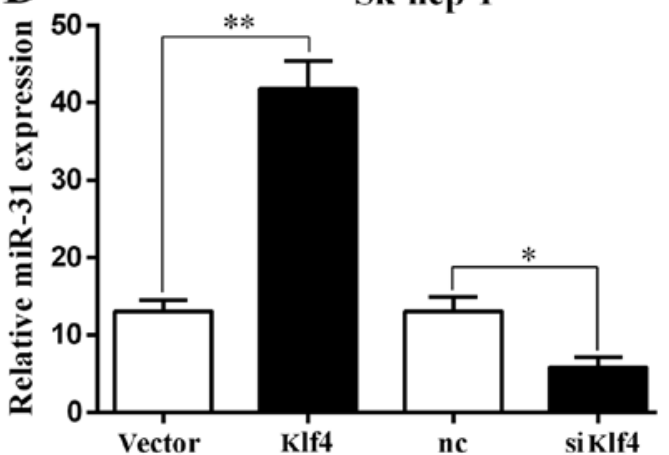

F

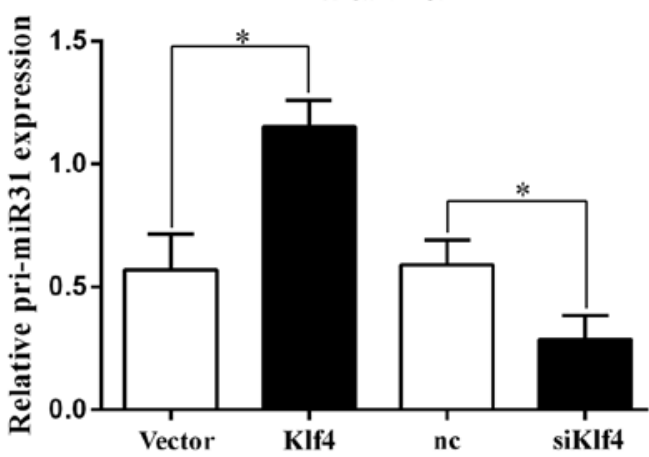

H

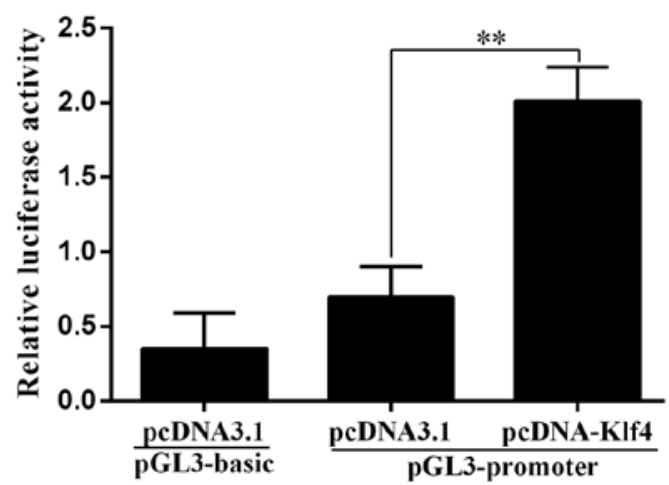

Figure 3. miR-31 is directly regulated by the transcription factor Kruppel-like factor 4 (K1f4). (A) RT-qPCR and western blot analysis of K1f4 expression in Sk-hep-1 and Bel-7402 cells transfected with Klf4 overexpression vector. (B-D) RT-qPCR analysis of miR-31 expression in Hep3B, Bel-7402 and SK-hep-1 cells transfected with Klf4 overexpression vector or siKlf4. (E-G) RT-qPCR analysis of pri-miR-31 expression in Hep3B, Bel-7402 and Sk-hep-1 cells transfected with Klf4 overexpression vector or siKlf4. (H) Relative luciferase activity of the miR-31 promoter in Hep3B cells transfected with pcDNA-Klf4. Luciferase constructs containing the miR-31 promoter (pGL3-promoter) and the unmodified construct (pRL-TK) were co-transfected with pcDNA3.1 and pcDNA-K1f4 into Hep3B cells. Firefly luciferase activity was normalized to Renilla luciferase activity. ${ }^{*} \mathrm{P}<0.05 ;{ }^{* *} \mathrm{P}<0.01$. 

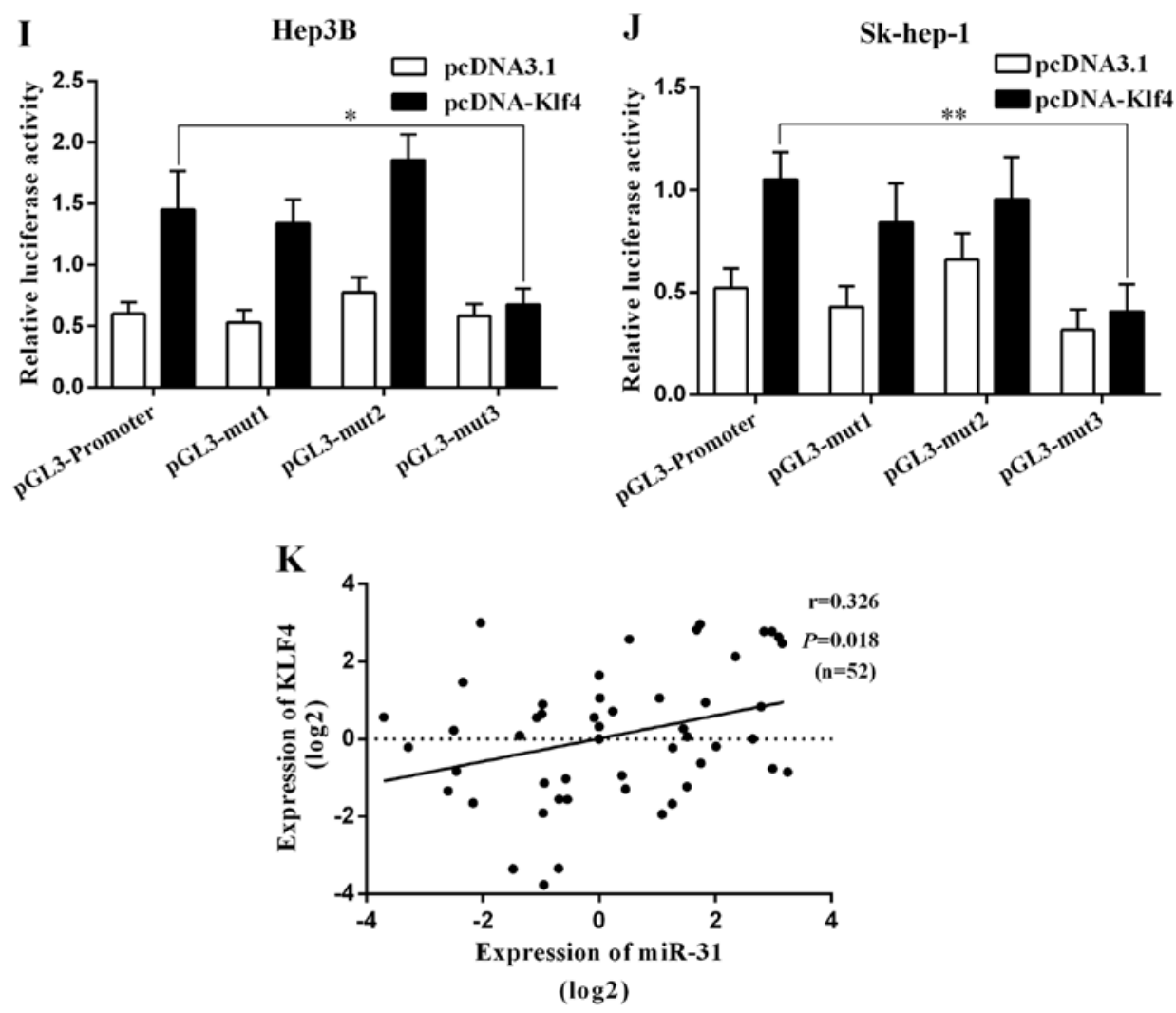

Figure 3. Continued. (I and J) Relative luciferase activity of the mutant miR-31 promoter constructs in Hep3B and Sk-hep-1 cells transfected with pcDNA-Klf4. Firefly luciferase activity was normalized to Renilla luciferase activity. (K) Klf4 mRNA expression positively correlated with miR-31 in 52 pairs of hepatocellular carcinoma (HCC) patients using linear regression models. The values of miR-31 were presented by the $\log _{2}$-fold change (HCC/NT). The data represent the means \pm SD from 3 independent experiments. ${ }^{*} \mathrm{P}<0.05 ;{ }^{* *} \mathrm{P}<0.01$.

every $24 \mathrm{~h}$. Mimic NC and inhibitor NC represents negative control miRNA.

As shown in Fig. 4C, transfection with miR-31 mimic decreased the number of Sk-hep-1 migrating cells, which had been increased by transfection with siKlf4. Similarly, transfection with miR-31 inhibitor increased the number of Bel-7402 migrating cells, which had been decreased by transfection with Klf4 overexpression vector. Consistent with the Transwell assay results, following transfection with the miR-31 mimic, the Sk-hep-1 cells migrated more slowly than the controls and the siKlf4-transfected cells, as shown by wound healing assays (Fig. 4D). By contrast, the Bel-7402 cells transfected with miR-31 inhibitor exhibited a more rapid migration rate compared with the controls and the cells transfected with the Klf4 overexpression plasmid, as shown by wound healing assays (Fig. 4D). These results demonstrate that miR-31 contributes to the regulation of liver cancer cell motility and progression.

\section{Discussion}

Over the past decade, the aberrant expression of miRNAs has been increasingly reported in human cancers (5). Numerous studies have shed light on tumor-targeting therapies using miRNAs as a novel diagnostic and therapeutic tools $(7,8,12,25,28)$. In the present study, the downregulation of Klf4 and miR-31 was consistently observed in HCC tissues and they may thus serve as potential diagnostic markers and therapeutic targets for HCC. Furthermore, the ectopic expression of miR-31 suppressed HCC cell proliferation and migration which was increased by transfection with siKlf4 in vitro. Moreover, the transcription factor Klf4 regulated the expression of miR-31, which in turn affected the progression of HCC. Our data suggest an important role of miR-31 in the progression of HCC.

Klf4 is a zinc finger transcription factor. Previous studies have indicated that Klf4 expression is reduced in different types of cancer $(29,30)$. Accumulating clinical evidence also suggests that Klf4 functions as a tumor suppressor gene $(31,32)$. Klf4 knockdown has been shown to promote cell growth, migration and adhesion, while Klf4 overexpression inhibits the proliferation of HCC cells (27). This study revealed that Klf4 was decreased in liver cancer tissues and cells compared with normal liver tissues. Therefore, the low expression of Klf4 in liver cancer may contribute to the reduced expression of miR-31 and may also play a crucial role in the progression of HCC.

miRNAs are post-transcription regulators of many genes, and their deregulation is related to cancer initiation, development and progression (33). Proliferation and metastasis, two hallmarks of malignancy, are the leading causes for cancerrelated death (34). It has been demonstrated that miRNAs are associated with these two events. For example, it has been shown that miR-17-5p promotes HCC cell migration and proliferation by targeting the p38-HSP27 pathway (35), and miR-26a has been shown to inhibit HCC growth and metastasis by suppressing IL-6 (10). In the present study, we demonstrated that miR-31 expression was decreased in liver cancer tissues and cells. However, the underlying mechanisms involved in 

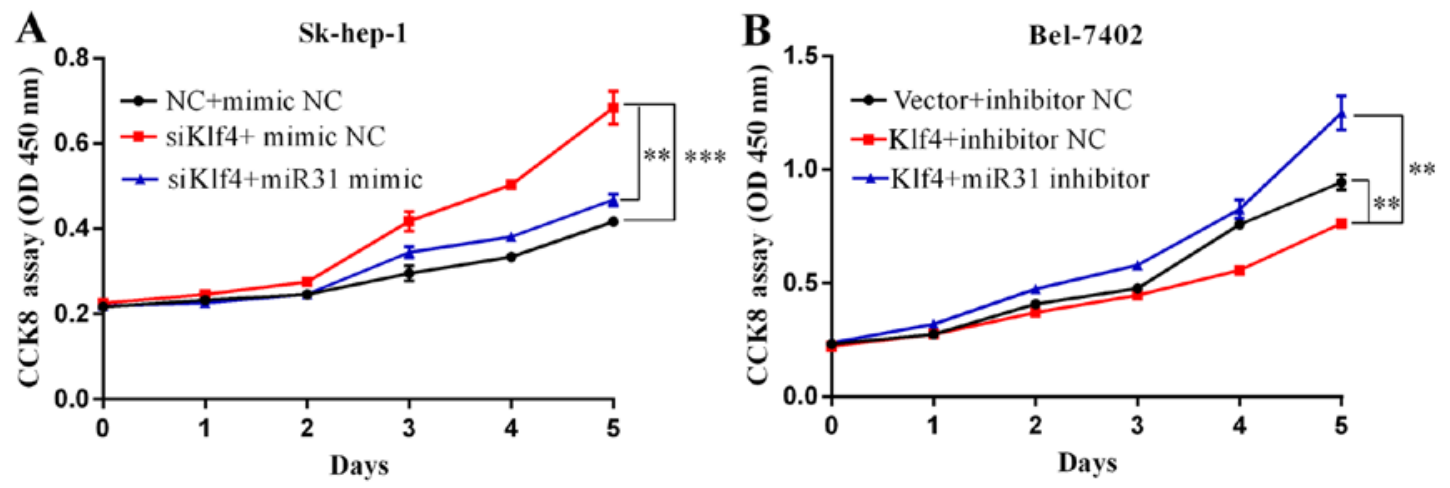
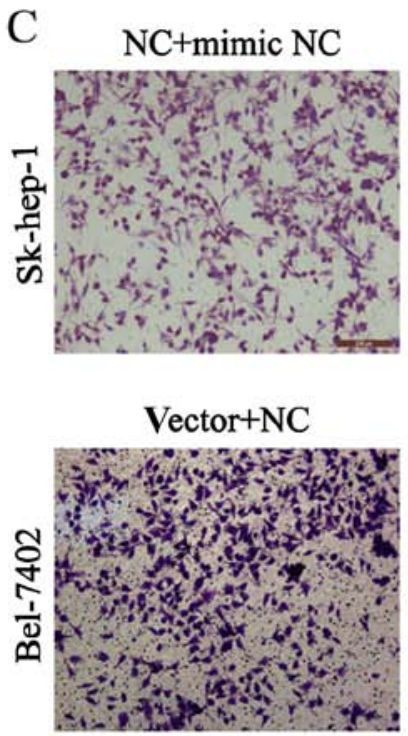

siKlf4+mimic NC

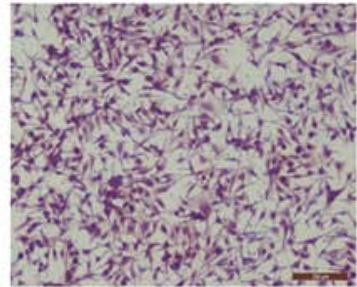

$\mathrm{Klf} 4+\mathrm{NC}$

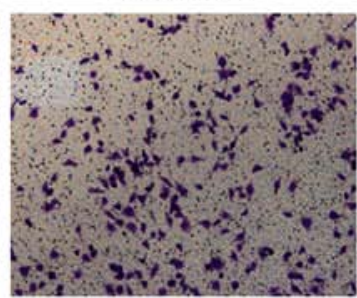

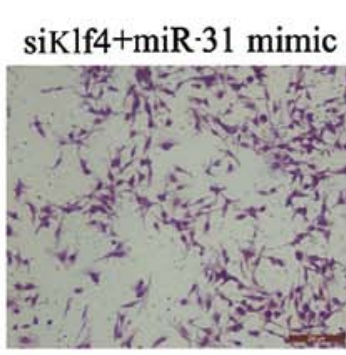

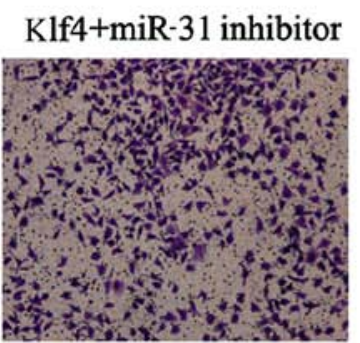

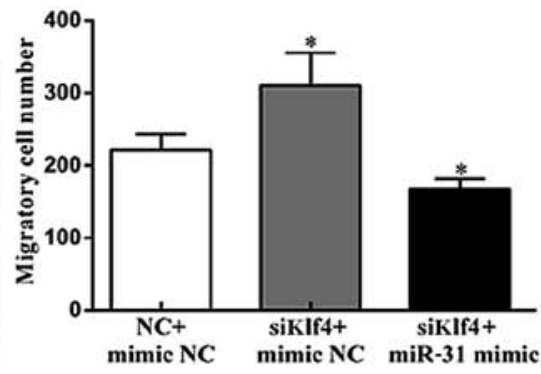

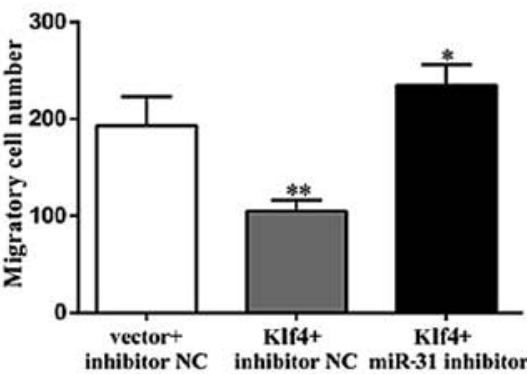

$\mathrm{D}$

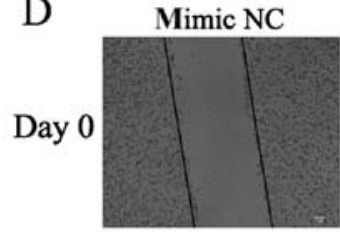

siKlf4+mimic NC

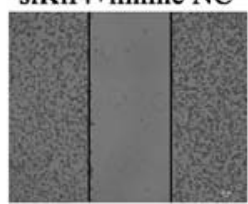

Day 2
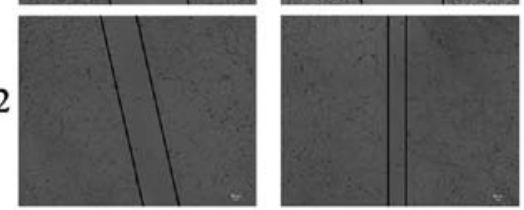

Vector+inhibitor NC
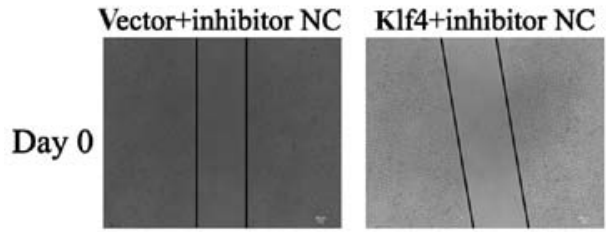

Day 2

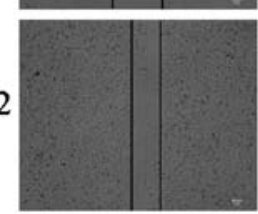

siKlf4+miR31 mimic
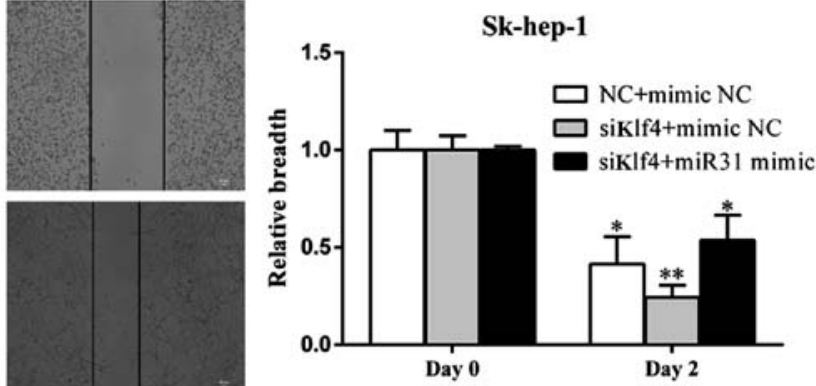

Bel-7402

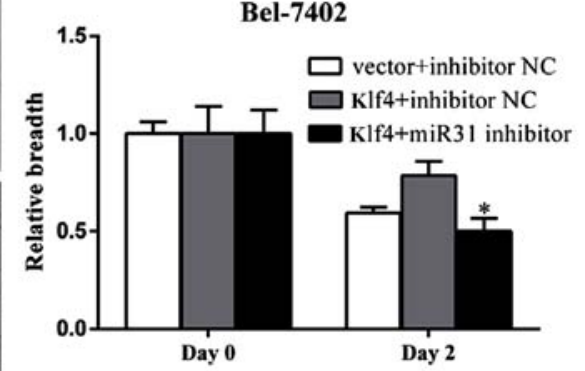

Figure 4. Kruppel-like factor 4 (Klf4) and miR-31 inhibits hepatocellular carcinoma (HCC) cell proliferation and migration in vitro. (A) Transfection with siKlf4 promoted Sk-hep-1 cell proliferation. Transfection with miR-31 mimic decreased cell proliferation in the cells transfected with siKlf4. (B) Klf4 overexpression suppressed the proliferation of Bel-7402 cells transfected with miR-31 inhibitor. Cells were seeded onto 96-well plates and transfected with Klf4 overexpression vector or miR-31 mimic for 5 days. Cell viability was determined by CCK8 assay. Cell growth was measured every 24 h. Mimic NC or inhibitor NC represents the negative control miRNA. (C) Transwell motility assays of Sk-hep-1 and Bel-7402 cells transfected with siKlf4 and miR-31 mimic or Klf4 overexpression vector and miR-31 inhibitor. The number of migrated cells was determined in randomly selected fields and is presented in the bar graph. (D) Wound healing assays were performed to investigate the mobility of liver cancer cells indicated (means $\pm \mathrm{SD} ;{ }^{*} \mathrm{P}<0.05 ;{ }^{* *} \mathrm{P}<0.01$ and ${ }^{* * * *} \mathrm{P}<0.001$ compared with the control, as shown by the Student's t-test). 
the deregulation of miR-31 are still unknown. Therefore, we investigated the correlation between the nuclear transcription factor Klf4 and miR-31 in human HCC and its effect on liver cancer cell proliferation and metastasis.

Collectively, our results provide experimental evidence that the transcription factor Klf4 suppresses cell growth and metastasis by directly targeting miR-31 in HCC. The newly identified $\mathrm{Klf} 4 \mathrm{miR}-31$ signalling axis may provide new insight into the pathogenesis of HCC and represents a potential therapeutic target for the treatment of HCC.

\section{Acknowledgements}

The present study was supported in part by grants from the National Natural Science Foundation of China (nos. 81272714, 81572310 and 81472576) and the KeyDisciplines Group Construction Project of Pudong Health Bureau of Shanghai (no. PWZxq2014-04).

\section{References}

1. Maluccio M and Covey A: Recent progress in understanding, diagnosing, and treating hepatocellular carcinoma. CA Cancer J Clin 62: 394-399, 2012.

2. Bhayani NH, Jiang Y, Hamed O, Kimchi ET, Staveley$\mathrm{O}^{\prime}$ Carroll KF and Gusani NJ: Advances in the pharmacologic treatment of hepatocellular carcinoma. Curr Clin Pharmacol 10: 299-304, 2015.

3. Moeini A, Cornellà $H$ and Villanueva A: Emerging signaling pathways in hepatocellular carcinoma. Liver Cancer 1: 83-93, 2012.

4. Lujambio A and Lowe SW: The microcosmos of cancer. Nature 482: 347-355, 2012.

5. Di Leva G, Garofalo M and Croce CM: MicroRNAs in cancer. Annu Rev Pathol 9: 287-314, 2014.

6. Fang W and Bartel DP: The menu of features that define primary microRNAs and enable de novo design of dicroRNA genes. Mol Cell 60: 131-145, 2015.

7. Parpart S, Roessler S, Dong F, Rao V, Takai A, Ji J, Qin LX, Ye QH, Jia HL, Tang ZY and Wang XW: Modulation of miR-29 expression by $\alpha$-fetoprotein is linked to the hepatocellular carcinoma epigenome. Hepatology 60: 872-883, 2014.

8. Najafi Z, Sharifi M and Javadi G: Degradation of miR-21 induces apoptosis and inhibits cell proliferation in human hepatocellular carcinoma. Cancer Gene Ther 22: 530-535, 2015.

9. He XX, Guo AY, Xu CR, Chang Y, Xiang GY, Gong J, Dan ZL, Tian DA, Liao JZ and Lin JS: Bioinformatics analysis identifies miR-221 as a core regulator in hepatocellular carcinoma and its silencing suppresses tumor properties. Oncol Rep 32: 1200-1210, 2014.

10. Yang X, Liang L, Zhang XF, Jia HL, Qin Y, Zhu XC, Gao XM, Qiao P, Zheng Y, Sheng YY, et al: MicroRNA-26a suppresses tumor growth and metastasis of human hepatocellular carcinoma by targeting interleukin-6-Stat 3 pathway. Hepatology 58 $158-170,2013$

11. Baek S, Cho KJ, Ju HL, Moon H, Choi SH, Chung SI, Park JY, Choi KH, Kim Y, Ahn SH, et al: Analysis of miRNA expression patterns in human and mouse hepatocellular carcinoma cells. Hepatol Res 45: 1331-1340, 2015.

12. Mlcochova J, Faltejskova-Vychytilova P, Ferracin M, Zagatti B, Radova L, Svoboda M, Nemecek R, John S, Kiss I, Vyzula R, et al: MicroRNA expression profiling identifies miR-31-5p/3p as associated with time to progression in wild-type RAS metastatic colorectal cancer treated with cetuximab. Oncotarget 6 : 38695-38704, 2015.

13. Tseng SH, Yang CC, Yu EH, Chang C, Lee YS, Liu CJ, Chang KW and Lin SC: K14-EGFP-miR-31 transgenic mice have high susceptibility to chemical-induced squamous cell tumorigenesis that is associating with $\mathrm{Ku} 80$ repression. Int J Cancer 136: 1263-1275, 2015.

14. Lu WC, Liu CJ, Tu HF, Chung YT, Yang CC, Kao SY, Chang KW and Lin SC: miR-31 targets ARID1A and enhances the oncogenicity and stemness of head and neck squamous cell carcinoma. Oncotarget: Aug 9, 2016 (Epub ahead of print).
15. Gao W, Liu L, Xu J, Shao Q, Liu Y, Zeng H and Shu Y: A systematic analysis of predicted MiR-31-targets identifies a diagnostic and prognostic signature for lung cancer. Biomed Pharmacother 68: 419-427, 2014.

16. Taccioli C, Garofalo M, Chen H, Jiang Y, Tagliazucchi GM, Di Leva G, Alder H, Fadda P, Middleton J, Smalley KJ et al: Repression of esophageal neoplasia and inflammatory signaling by anti-miR-31 delivery in vivo. J Natl Cancer Inst 107: djv220, 2015.

17. Wang S, Li Q, Wang K, Dai Y, Yang J, Xue S, Han F, Zhang Q, Liu J and Wu W: Decreased expression of microRNA-31 associates with aggressive tumor progression and poor prognosis in patients with bladder cancer. Clin Transl Oncol 15: 849-854, 2013.

18. Schaefer A, Jung M, Mollenkopf HJ, Wagner I, Stephan C, Jentzmik F, Miller K, Lein M, Kristiansen G and Jung K: Diagnostic and prognostic implications of microRNA profiling in prostate carcinoma. Int J Cancer 126: 1166-1176, 2010.

19. Ruoming W,Zhen $Y$, Tengteng $Z$ and Jisheng $H$ : Tumor suppressor microRNA-31 inhibits gastric carcinogenesis by targeting Smad4 and SGPP2. Cancer Gene Ther 22: 564-572, 2015.

20. Kim HS, Lee KS, Bae HJ, Eun JW, Shen Q, Park SJ, Shin WC, Yang HD, Park M, Park WS, et al: MicroRNA-31 functions as a tumor suppressor by regulating cell cycle and epithelialmesenchymal transition regulatory proteins in liver cancer. Oncotarget 6: 8089-8102, 2015.

21. Viré E, Curtis C, Davalos V, Git A, Robson S, Villanueva A, Vidal A, Barbieri I, Aparicio S, Esteller M, et al: The breast cancer oncogene EMSY represses transcription of antimetastatic microRNA miR-31. Mol Cell 53: 806-818, 2014.

22. Creighton CJ, Fountain MD, Yu Z, Nagaraja AK, Zhu H, Khan M, Olokpa E, Zariff A, Gunaratne PH, Matzuk MM and Anderson ML: Molecular profiling uncovers a p53-associated role for microRNA-31 in inhibiting the proliferation of serous ovarian carcinomas and other cancers. Cancer Res 70: 1906-1915, 2010.

23. Zhang N, Zhang J, Shuai L, Zha L, He M, Huang Z and Wang Z: Krüppel-like factor 4 negatively regulates $\beta$-catenin expression and inhibits the proliferation, invasion and metastasis of gastric cancer. Int J Oncol 40: 2038-2048, 2012.

24. Li Q, Gao Y, Jia Z, Mishra L, Guo K, Li Z, Le X, Wei D, Huang S and Xie K: Dysregulated Krüppel-like factor 4 and vitamin D receptor signaling contribute to progression of hepatocellular carcinoma. Gastroenterology 143: 799-810.e1, 2, 2012.

25. Yu T, Chen X, Lin T, Liu J, Li M, Zhang W, Xu X, Zhao W, Liu M, Napier DL, et al: KLF4 deletion alters gastric cell lineage and induces MUC2 expression. Cell Death Dis 7: e2255, 2016.

26. Lv H, Zhang Z, Wang Y, Li C, Gong W and Wang X: MicroRNA-92a promotes colorectal cancer cell growth and migration by inhibiting KLF4. Oncol Res 23: 283-290, 2016.

27. Liu S, Yang H, Chen Y, He B and Chen Q: Krüppel-like factor 4 enhances sensitivity of cisplatin to lung cancer cells and inhibits regulating epithelial-to-mMesenchymal transition. Oncol Res 24: $81-87,2016$

28. Gandellini P, Giovannetti E and Nicassio F: MicroRNAs in cancer management: Big challenges for small molecules. Biomed Res Int 2015: 982156, 2015.

29. Zhou J, Lai PB and Tsui SK: Identification of a non-coding KLF4 transcript generated from intron retention and downregulated in human hepatocellular carcinoma. Int J Oncol 47: 1554-1562, 2015.

30. Tien YT, Chang MH, Chu PY, Lin CS, Liu CH and Liao AT: Downregulation of the KLF4 transcription factor inhibits the proliferation and migration of canine mammary tumor cells. Vet J 205: 244-253, 2015.

31. Mao Q, Quan T, Luo B, Guo X, Liu L and Zheng Q: MiR-375 targets Klf4 and impacts the proliferation of colorectal carcinoma. Tumour Biol 37: 463-471, 2016.

32. Lin ZS, Chu HC, Yen YC, Lewis BC and Chen YW: Krüppel-like factor 4, a tumor suppressor in hepatocellular carcinoma cells reverts epithelial mesenchymal transition by suppressing slug expression. PLoS One 7: e43593, 2012.

33. Fiorenza A, Lopez-Atalaya JP, Rovira V, Scandaglia M, Geijo-Barrientos E and Barco A: Blocking miRNA biogenesis in adult forebrain neurons enhances seizure susceptibility, fear memory, and food intake by increasing neuronal responsiveness. Cereb Cortex 26: 1619-1633, 2016.

34. Meehan K and Vella LJ: The contribution of tumour-derived exosomes to the hallmarks of cancer. Crit Rev Clin Lab Sci 53: 121-131, 2016.

35. Yang F, Yin Y, Wang F, Wang Y, Zhang L, Tang Y and Sun S: miR-17-5p Promotes migration of human hepatocellular carcinoma cells through the p38 mitogen-activated protein kinase-heat shock protein 27 pathway. Hepatology 51: 1614-1623, 2010. 